\title{
The Operational Present of Sensibility
}

\author{
Mark B. N. Hansen
}

\begin{abstract}
Contemporary digital technologies afford unprecedented access to levels of temporal experience that have long remained beyond the scope of human thematization. In their efforts to historicize these affordances, historians of science have insisted on the peculiarity of this access, the fact that it does not take place through any direct expansion of human perceptual capacities, but rather through a human-machine assemblage that supplements perception by putting it into systemic co-relation with a technical operationality whose "content" perception cannot access. In this article, Hansen utilizes this indirect model of the temporal expansion of human agency to explore contemporary micro-computational expansion of sensibility, both as it informs data capitalism and efforts to counter the latter's sway.

KEYWORDS Perception, human-machine assemblage, technical operationality, data capitalism
\end{abstract}

\section{The Precognitive Vocation of Twenty-First Century Media}

"With ever-expanding volumes of stored data to draw upon, and new ways of connecting people, machines and forces - distributing and sharing their functions in a larger field of human and machinic agency - relationships are uncovered among widely disparate kinds of information. Through a technologically enhanced perception, a mathematical seeing, patterns come into view that previously could not be seen by the naked eye, in ways that augment, or occlude, traditional observational expertise and human intuition." ${ }^{1}$ With this account of the contemporary technical distribution of precognitive sensibility, media artist and theorist Jordan Crandall perfectly captures both the vastly expanded sensory field within which contemporary events occur and the fine-tuning of our access to the separate, most often microtemporal performances, both machinic and human, that contribute to their occurrence.

What is distinctive about Crandall's account, and what motivates my invocation of it at the beginning of my talk, is his willingness to associate the technical transformations that lie at the heart of twenty-first century media - and that witness a fullscale installation of a calculative ontology of prediction - with distinct modifications in the structure of experience. He rightly discerns that technological media are in no sense simply and solely technical, but are indelibly and inseparably technical, performative, affective, experiential, and sensory. Among Crandall's various characterizations of the broad correlations between media technicity and experience, most fundamental for my purposes is his keen insight into 
the de-coupling of effect and awareness - of causal efficacy and presentational immediacy to use philosopher Alfred North Whitehead's felicitous terms - that characterizes the operationality of contemporary media. What accounts for the singularity of contemporary media is not simply that their data-driven operations bypass the scope of consciousness, but that they impact experience on a much broader basis than consciousness. They literally seep into the texture of experience, forming a background, a peripheral "calculative ambience," that indirectly flavors any and all resulting events or phenomena:

As tracking becomes elevated into a condition, dissolving into behavior, sensation and all manner of embodied social practices in the data-intensive, analytics-driven spaces of megacities, the 'sense of continual access to information' that arises out of the connectivity and interoperability among all kinds of data-enhanced actors (Thrift 2008: 92-99) is not necessarily grounded in a direct access. It is not simply a matter of whether one has a direct connection to this data-intensive surround, since it increasingly constitutes a defining horizon against which the phenomena of the megacity are understood - a calculative ambience that imposes its distinction, categories and ways of being onto all facets of urban life - as it acts as a cognitive, ontological, and experiential supplement for the simplest forms of ordinary routine. ${ }^{2}$

With his appreciation for the supplementarity of twenty-first century media's experiential impact, and for the curious logic whereby what is supplementary becomes more fundamental than what it supplements, Crandall's analysis directly addresses the experiential paradigm catalyzed into operation by twenty-first century media. In particular, it allows us to appreciate that the data-intensive, analytics-driven media surround operative in the contemporary urban environment impacts us not by affording direct, cognitive access to information, but rather by creating a tacit atmosphere of sensibility for action and capacities for datagathering and analysis that open possibilities for precognitive shaping of - and capture of information about - our actions or likely tendencies for action.

Crandall's analysis underscores how much the experiential paradigm of twenty-first century media results from an increasingly sophisticated and ubiquitous technical capacity to gather data concerning aspects of experience that are not directly accessible to us qua individual agents, that we simply cannot experience through consciousness and perceptual awareness. In this sense, twenty-first century media is characterized first and foremost by the capacity for capturing information that directly 
concerns our behavior and tendencies but to which we ourselves lack any direct access. This fundamental separation of data-gathering from experience forms the basis for what I shall theorize, with Crandall and others, as the precognitive vocation of twenty-first century media. This vocation stems from the tendency of twenty-first century media to target the infrastructural or causally efficacious elements informing future behavior with the aim of reliably predicting such behavior before it actually happens.

\section{Media and Capital}

The precognitive vocation of twenty-first century media is deeply imbricated with the operation of global capital. Following the advent of network capitalism, the extraction of surplus value that is a generic feature of capitalism has increasingly come to focus on the value of data, as it were, "automatically" gathered from traces left by our living activity. A broad range of theorists, notably including Maurizio Lazzarato, Tiziana Terranova, and Franco "Bifo" Beradi, have reflected on how forms of immaterial labor have, with the acceleration of internet culture, transformed into free labor: the production of content that, in a closed yet infinitely expanding spiral, serves as lure for further internet traffic, and with it the production of more value. Post-Autonomist philosopher Matteo Pasquinelli, writing about Google's PageRank algorithm, invokes the term "network surplus value" to distinguish this shift in the locus of value. Itself an operation on twenty-first century media, network surplus value transforms data about human behavior that is not accessible to human awareness into value. That is why geographer Nigel Thrift can claim that contemporary capitalism operates first and foremost by exploiting its marginal advantage in manipulating this sub-perceptual data:

What is new about the current conjuncture is the way in which capitalism is attempting to use the huge reservoir of non-cognitive processes, of forethought, for its own industrial ends in a much more open-ended way. ... more recently, much thought has been given to understanding forethought as not just a substrate but as a vital performative element of situations, one which cannot only produce its own intelligibilities but which can be trained to produce ideas. ... now the intention is to read and exploit signs of invention by regarding the body as a mine of potentiality and to generate and harness unpredictable interactions as a source of value .... ${ }^{3}$

Thrift's account of contemporary data capitalism foregrounds the temporal dimension of its operation: if capitalist institutions have succeeded 
in exploiting the precognitive processes of bodily life that generate "forethought," they have done so precisely because they have found ways to access these processes in their own "operational" timeframe. Today's culture industries can tap the bodily processes leading to "foresight" - the domain of Whitehead's causal efficacy - because they are able, with the help of microcomputational sensors, to access the sensory output of these processes independently of the normal embodied circuits that, via some kind of emergence, yield forethought as a bodily feeling, as affective anticipation. It is as if microcomputational sensors literally wrested these processes from their natural embodied context and made them independently operable and accessible.

What independent access in this case affords is a capacity to manipulate "forethought" as a technical variable, and crucially, to exploit its value in timeframes that are far closer to the operational timeframe of the precognitive embodied processes than to the operational timeframe of whatever cognitive output - "forethought" in its proper sense - emerges when they are processed by the body. To the extent that the timeframes at issue here are smaller than those of bodily emergence, and are smaller - by an order of magnitude - than the time of consciousness, this operation is one that bypasses consciousness, and that is literally premised upon the bypassing of consciousness.

Because it grasps the correlation between data-intensive media and experience in its full potentiality, a theorization of twenty-first century media like the one I have been developing here in itself rounds out the picture that capitalist institutions would like to truncate. Theorization of twenty-first media shows that the sway exercised by contemporary capitalist institutions is a function of their capacity to control the time of experience and is thus a site for potential political struggle. Contemporary capitalist industries are able to bypass consciousness - and thus to control individual behavior - precisely because of their capacity to exploit the massive acceleration in the operationality of culture generated by massive-scale data gathering and predictive analysis. These industries benefit from the maintenance of the crucial temporal gap at the heart of experience: the gap between the operationality of media and the subsequent advent of consciousness.

We can get a sense for the temporal structure at issue here by comparing it with the microtemporal gap separating neuronal events from consciousness as neuroscientists like Libet and Damasio understand it. In both cases, consciousness arises with a constitutive delay - Libet's "missing half-second" - in relation to its causal efficacy (and irregard- 
less of whether that efficacy stems from neural processing or from data gathering). Thus, where neuroscience questions the agency of consciousness, asking ultimately whether we have free will at all if consciousness is limited to the function of ratifying what has already been decided, twenty-first century media effectively represses consciousness by operating in technical timeframes to which consciousness has absolutely no direct, experiential or phenomenological interface. Within this situation, as I hope to show a bit later on, consciousness is generated after-the-fact, as an emergence generated through the feeding forward of technicallygathered data concerning antecedent microtemporal events.

\section{The Braintime Assemblage}

Indeed, as historian of science Henning Schmidgen has compellingly argued, the phenomenon of the missing half-second results from - and can be sustained only through - the construction of a "cyborg assemblage" in the laboratory. ${ }^{4}$ Far from being a faculty of (human) experience, which is how Thrift, Brian Massumi, and other contemporary affect theorists effectively treat it, the missing half-second is an artifactual construction that has no agency independently of its technical set-up - that, in short, has no "natural" agency whatsoever. Not only does this mean that the missing half second cannot exist as a self-standing agency, independently of the technical apparatus facilitating its presentification, but it also means that it cannot directly function to intensify or granulate sensing and perceiving. There simply is no direct phenomenological interface onto the operation of braintime.

When theorists thematize the missing half second as a "space of action," as Thrift does, or as an expanded form of "thinking-feeling," as Massumi does, or finally as a "pre-acceleration" of perception, as Erin Manning does, they all make the mistake of ignoring the technical, social, and material specificity of the "braintime" experiment and the laboratory assemblage that underlies it. In effect, these theorists abstract from the concrete linkages that are necessary to materialize the missing half second as a phenomenon. When they subsequently treat this abstracted phenomenon as an expanded space of action for human agency - the space where, say, affectivity operates before the emergence of cognition - they indulge in a fantasy that, from Schmidgen's perspective (and I whole-heartedly concur), is utterly groundless. We humans simply do not have access to the space of the missing half second in any situation other than that of the braintime assemblage. Thus, contra Thrift, contemporary neural imaging technologies do not and cannot "open up" the "small space of time ... 
which shapes the moment" for apprehension in sense perception, though they do open it for targeted marketing by contemporary data industries. What Thrift, Massumi, and Manning all ignore - and what Schmidgen's work helps to underscore - is the very important fact that the technical presentification of the missing half second is from its very onset an originarily technical artifact. Or, to put it another way, they ignore the fact that the missing half second becomes accessible to us only as data, and indeed, as data about experience of which we can have absolutely no awareness. The missing half-second is impervious to phenomenological inspection, which is precisely why it - or rather the data gathered about it - is of great interest to today's data industries: for the same reason that it is an "originally" technical artifact, the data of the missing half-second is, as it were, originarily available to be "operated on" by the "fastest bidder," by whatever institution can access it most quickly.

Of the contemporary theorists interested in affect and the emotional brain, it is perhaps the philosopher Catherine Malabou who begins to sketch out how humans might "experience" their own neuronal processes, their braintime. With her crucial notion of the "cerebral unconscious," Malabou depicts the brain as a radical exteriority, a kind of alien being, lodged within the psyche: crucially, the cerebral unconscious remains open to the radical or destructive, and radically exterior, accident. Unlike Freud's sexual unconscious, this cerebral unconscious makes the brain into an access point onto an exteriority - and potentially on the exteriority of data - that cannot be treated as endogenous, and cannot be mastered by the hermeneutic protocols of Freudian theory. What ultimately emerges from this notion is a new account of auto-affection: an autoaffection that is always necessarily absent from the present of consciousness and that is always in relation to a radical outside:

The nature of cerebral auto-affection is different than the auto-affection of the subject as the philosophers have defined it. The elementary reflection that constitutes the cerebral psyche as such does not reflect upon itself. It does not redouble its specularity to the point of endowing it with the form of consciousness. No one can feel his or her own brain; nor can he or she speak of it, hear it speak, nor hear himself or herself speak within it. Cerebral auto-affection is necessarily and paradoxically accompanied by a blindness, an inability of the subject to feel anything as far as it is concerned. ${ }^{5}$

Such an autoaffective brain is "only accessible by means of cerebral imaging technology. And there is no possible subjectification of this type of objectification." 
But let me return to the originary technicity of braintime. Although capacities for manipulating the space of the missing half second have multiplied expontentially in the wake of digital computation, the structure of its operation has remained constant since the mid-19th century when the protocol of what Schmidgen calls the "braintime experiment" was initially configured: this protocol involves using technical acceleration to access psycho-physiological processes imperceptible to "natural" perception in order to slow them down for subsequent perceptual apprehension. ${ }^{7}$ What is true for these psycho-physiological processes - namely, that they cannot constitute agencies independently from the technical set-up that makes them accessible - is all the more true for the domain of sensibility opened up by twenty-first century media. That is why what we learn from the longer history of the braintime experiment can help us to appreciate what is involved in the industrial co-optation of sensibility: rather than operating on the basis of a gap within our "natural" perceptual faculty, a gap between neural processing and subsequently emergent mental phenomena, this co-optation, as shown by the protocol of the braintime experiment, operates on a gap between our "natural" perceptual faculty and the radically exterior domain of sensibility.

We can get a clear sense for the stakes involved here by invoking the contemporary perversion of the pharmacological imperative of technics. Rooted in Plato's conception of writing as both a poison (pharmakon) and its own antidote or remedy, pharmacology has been developed by Derrida, Bernard Stiegler, Avital Ronnell and others, as a means of theorizing the impact of media technology on human life. The dominant pattern for such theorizing is to present media as pharmacological in the sense that it gives recompense for what it takes away. Indeed, this narrative pattern is deeply intertwined with Western thinking on media, as we can see in considering the widespread impact of McLuhan's conception of media amputation and expansion: for McLuhan, as for Derrida and Stiegler, what media takes away from the human bodymind is given back in the form of a prosthetic extension. However, I want to suggest that when our behavior becomes accessible as technically-generated data that we ourselves cannot experience directly, this prosthetic pharmacology undergoes a fundamental transformation. Put schematically, we could say that media pharmacology loses its prosthetic basis since the loss of our agency over our own behavioral data is recompensed by something that has no direct correlation with it, namely, the affordances of social media. I have elsewhere described this transformation as a splitting of the pharmacological structure of media that involves the production of an experi- 
ential level - the world of social media - as a false recompense for what is really going on, namely the extraction of "data-value" in the form of user profiles. What is particularly interesting about this splitting, and what merits the characterization of a "perversion," is the way it overlays a traditional pharmacological narrative (we give up our data and are given in return the affordances of social media) on a quite different story (we give up our data and are thereby transformed into commodities, data profiles, to be sold to the highest bidder).

We can get a clearer understanding for the nuances of this transformation by comparing social media with writing. Writing, as both Derrida and Paul de Man established in their work on figures like Plato and Hegel, involves the replacement of one form of memory (interior memory, or Erinnerung) with another (artifactual memory, or Gedächtnis). And despite the success of their deconstructive readings in revealing the dependence of interior memory on technical memory - which is also to say, the "originary technicity" of memory - there is a sense in which writing qua technical memory serves, or more exactly, has always served, as a recompense, and indeed as a phenomenological recompense, for the weaknesses of natural memory. Despite its superficial similarity to the pairing of interior and exterior memory, the pharmacological relationship at work in contemporary techniques for gathering data about what happens in the missing half-second - and in the braintime experiment - involves the deployment of technics to access and presentify data that is radically disjunctive with phenomenological modes of experience. For, whereas artificial memory aids as theorized by philosophers from Plato via Hegel to Stiegler technically exteriorize memory without changing the form of its content, technical access to data of sensibility and to the neural processes comprising braintime operate in lieu of any possible phenomenological mode of experience. Like Etienne-Jules Marey's graphic and chronophotographic machines which must be understood to be autonomous sensing agents that possess their own sensible domains, the cyborg assemblages that access and capture the data of sensibility do so in radical disjunction from any possible (future) presentification to perceptual consciousness. Accordingly, when this data is fed forward into our embodied experience, as I have argued it must be if it is to be experienced by us at all, it marks the intrusion of a radical exteriority into consciousness, an exteriority that cannot so much be interiorized as introjected.

This is why any attempt to grasp the technicity introduced by twentyfirst century media by way of affectivity - understood as a subperceptual but nonetheless still direct connection to sensibility - remains insuf- 
ficiently radical. It remains insufficiently radical because the technical capture of data of sensibility simply has and can have no direct experiential interface. Even those affective dimensions of experience that serve to prime ensuing perceptions can neither be directly experienced, nor capture the broader and more primordial interface with worldly sensibility that, as I have sought to demonstrate, comes before the consolidation of any subjective unity capable of hosting such affectivity. In full resonance with Whitehead's description of the "dative phase" of actualization, where the insistence of the immediately preceding state of the universe catalyzes the genesis of a new actuality, this interface generates a primordial feeling - a primal impressionality - that is preaffective, a feeling without a feeler.

\section{The Feed-Forward Structure of Twenty-First Century Consciousness}

For my purposes, a crucial factor differentiates the technical delay operated by twenty-first century media from the neuronal delay at issue in the braintime experiment- namely, its radical exteriority. The information captured by microsensors is accessed and fed forward into consciousness not as the material basis for an emergent mental state but, quite literally, as an intrusion from the outside. As such, it does far more than simply support emergent mental contents; indeed, it captures a far larger swathe of the causal efficacy supporting the behavior that underpins such emergent states, including much that simply cannot find its way into consciousness via any organization or assembly of neurons. In this way, the data gathered by technical inscription makes available to consciousness aspects of its own causal background that it literally has no capacity to grasp directly, by embodied pathways. And it does this by way of a temporal dynamics that is characterized by a fundamental futural orientation: rather than marking the essential correlation of our present experience with what is now past, or more precisely, just-past, as it does for the orthodox phenomenology of time-consciousness as well as for Whitehead's concept of "nonsensuous perception," this technically-constituted delay reorients everything to an almost present future moment in which present, "operational" experience becomes - or more precisely, will have become - available to consciousness and to further operations, including conscious reflection and deliberation and, importantly, targeted modification of embodied behavior. This is the "feed-forward" structure of twenty-first century consciousness.

Once again, Crandall seems to grasp the technico-experiential specificity of this temporal predicament: 
The Operational Present of Sensibility

As performatively constituted action-densities (Barad, 2007) inferred through calculative, predictive or pro-active operations, an actor integrates and internalizes, consolidates and extends within the organizational and ontological horizon of tracking - a field that harbors a fundamentally anticipatory orientation. Actors are characterized by what they $d o-$ instantiation is action - and what they do is inflected by what they will do. Actuality is conditioned by tendency. Embroiled in a calculative, mobilizing externality, agency pushes and is pulled outward, as if seeking to become the predisposition that it courts. ${ }^{8}$

By grasping how behavior resonates with its own futurity, Crandall foregrounds one essential element of the experiential paradigm introduced by twenty-first century media: the displacement of consciousness's function from direct awareness of experience as it is happening to a supervisory role that can be exerted only indirectly over future experience to come. Within the complex and heterogeneous fields of contemporary sensibility, consciousness undergoes a fundamental retooling: as its direct perception of its own causal efficacy gives way to a much expanded technical inscription of this same causal efficacy that can only be fed forward into, and thus indirectly experienced by, (a future) moment of consciousness, consciousness comes to learn that it lags behind its own efficacy. What consciousness experiences as its present - the present of sense perception - is, with respect to its efficacy, always already past. That, indeed, is precisely why Whitehead calls causal efficacy "nonsensous perception": by the time consciousness has sense perception of its experience, the causal basis for that perception will have become past, no longer sensuously present. Perception of causal efficacy is nonsensuous only because and only so long as it must be experienced through consciousness, only because and only so long as it cannot be experienced more directly, through alternate, technical channels.

\section{The Operational Present of Sensibility}

Everything changes, however, when we factor in the capacity of technical microsensors to capture data concerning causal efficacy. For if I am right that today's microcomputational sensors operate as sensing agents, then the data they generate about our behavior constitutes a form of worldly sensibility that marks a sensory present - what I have called the operational present - which we can now see is distinct from the present of consciousness. In light of the newfound capacity to directly register the present of sensory efficacity, and to feed this forward into conscious experience to come, the necessity to channel causal efficacy through the 
presentational immediacy of consciousness - the very necessity informing Whitehead's account of symbolic reference - would seem to dissolve. And what such a dissolution makes possible, we can now fully appreciate, is nothing short of a fundamental shift in the frame of reference that determines what counts as the sensory present: by directly capturing the sensory immediacy of causal efficacy in a form that is directly presentational (and that can be fed forward into future consciousness as an alternate, artifactual source of presentational immediacy), today's computational microsensors inaugurate the operation of a new level of presencing - the present of causal efficacy (what I shall call the operational present of sensibility) - that both supplements and, in a sense, takes over the longstanding role and privilege consciousness has exercised as agent of presencing. While consciousness continues to experience its own narrow bandwidth reality through sense perception, this experience is disjoined, both temporally and operationally, from the presencing of embodied causal efficacy - the operational present - where, increasingly, behavior gets operated on and controlled, independently of any conscious access or input. To the extent that contemporary technologies for data-gathering and analytics allow for predictive precognition of what is to come, they manage to define a microtemporal, subperceptual - yet still sensory - present that impacts the future independently of any input from consciousness. As a consequence of this refining of the operationality of the present, consciousness must trade in its former monopoly over presentation for a supervisory role: since its presentational immediacy always comes too late in relation to the operational present, consciousness must redirect its focus to a future that it can only experience indirectly. Rather than living its present as presentational immediacy (i.e., through sense perception), consciousness can only live it as a deferred or after-the-fact experience of technically-gathered sensory data concerning its own efficacy: consciousness literally encounters its own operationality only once this has been fed-forward, as artifactual presentification, into its imminent or looming future.

\section{The Ecological Pharmacology of Twenty-First Century Media}

Let me now explore the implications of this understanding of the operational present of sensibility for our theorization of media and media ecology. To do so, I want to return to the topic of pharmacology and underscore the possibility for a very different recompense for the loss of control over our behavioral data than that of social media affordances. To put it schematically, this recompense is the expanded sensory contact 
with the world that the technical revelation of the operational present of sensibility makes possible. Provided that we retain the right to access our own data and that we manage to develop technical assemblages for transforming such access into agency over the future, the technical incursion into the operational present of sensibility need not remain an opportunity exclusively available to today's data industries. Indeed, it has the potential to give us information about our own experience - information to which we have no phenomenal access - that can help us make our lives better in myriad ways.

Isn't this investment in the power of data to ameliorate our lives precisely the source for the appeal of the recent television drama, Person of Interest, in the sense that it features superhero-like characters who have imperfect knowledge of the predictions of an all-knowing but fully mysterious "machine" and who must act - and must embrace the uncertainties of acting - if they are to prevent predicted future murders? What Person of Interest dramatizes is a technical distribution of agency in which a highly sophisticated computational machine accesses data of worldly sensibility and feeds it forward into human experience. Unlike the film Minority Report (and even more so Philip K. Dick's short story "The Minority Report" on which it is based), there is in Person of Interest no possibility for an existential moment of self-recognition where one can modify one's "precognized" fate; rather, the characters "blindly" follow the clue furnished by the machine until they can, by acting in the near future-oriented present, figure out how the person identified by that clue is involved in a future murder and act to prevent its occurrence. Gone here is any hope for a reconciliation of the knowledge afforded by data (the precog's visions of the near future) and the knowledge afforded by experience: the "numbers" generated by the machine, which correspond to identities of persons, brook no interpretation, but function simply to trigger an action-based process of search that never fails to yield the desired goal of preventing murder.

What makes Person of Interest so resonant with my general claim concerning the expanded sensibility brokered by twenty-first century media is its taken-for-granted embrace of the "machine" as a sophisticated cognitive agent whose workings remain absolutely inscrutable to humans - indeed, unquestionably beyond human exploration as such. From what little we are told about the machine, we know that it processes massive amounts of video surveillance and cell phone data - the passive data that lies at the heart of twenty-first century media - in order to make predictions concerning the future. By depicting a co-functioning between the 
machine and the characters acting on its prediction, Person of Interest allegorizes the very condition I am seeking to theorize: the imperative for us to embrace the qualified marginalization of consciousness that goes together with any opportunity we might have to benefit from the technical access to the operational present of sensibility. Indeed, with its various compensatory narratives - one episode, for example, involves the salvation of a post-9/11 War Veteran whose guilt over the death of a fellow soldier and desire to support his family has led him to commit multiple armed robberies - Person of Interest could well be read as an allegory of the pharmacological recompense of twenty-first century media: by depicting the use of data extracted from human activity, not as a new source of economic value, but as the basis for superhero-like doinggood, the show capitalizes on the potential - and the popular desire - for cold, quantitative, dare I say "inhuman" data to benefit human life.

Person of Interest expresses the very imperative that we face in adapting to the networked regimes of twenty-first century media: it perfectly expresses how the pharmacological recompense specific to twenty-first century media requires an embrace of the very marginalization of consciousness that comprises the strategy of today's data industries. No longer can we take up embodiment as a site where diffuse data is processed to yield images or experiences, as I myself have argued in my previous work; rather, in the face of technical incursions that render the body directly "readable" by machines, we must embrace a conception of the body as a society of microsensibilities themselves atomically susceptible to technical capture.

Accordingly, whatever pharmacology we might develop on the basis of the affordances of twenty-first century media must break with the notion central to the prosthetic tradition of pharmacological through - namely that media's recompense comes in the form of some expansion of our embodied capacities that can be experienced as such by us. In marked contrast with this vision, the first principle of an alternate, ecological pharmacology must be the renunciation of any hope that we can operate - that we have any agency whatsoever - in the operational present of sensibility. Despite its seeming simplicity, this renunciation remains a challenge in that it cuts against all of the ingrained habits of thought we have developed to understand our experience, including the habit that tells us we can or should understand our experience. A case in point comes by way of the difficulties Nigel Thrift encounters in seeking to develop his own keen insight into the properly post-phenomenological realities of a world dominated by twenty-first century media. Renunciation of our 
capacity to act in the operational present of sensibility would seem to follow from Thrift's analysis of the "giant temporal shortcut" that informs contemporary strategies of neuro-marketing; as Thrift sees it, neuromarketing aims to substitute a simulated now content for the now of lived experience. Yet the fact that Thrift himself remains unable to draw this conclusion only attests to the powerful hold exerted by the fantasy of action in the missing-half second that I diagnosed above. Adapted for our contemporary moment, Schmidgen's work on the braintime assemblage operates as a kind of therapy: specifically, it helps us appreciate why we must embrace the displacement of phenomenological agency in favor of technical processing if we are to access the operationality of sensibility and tap into its compensatory potential. The preaffective present, what I have been calling the operational present of sensibility, can only be accessed as a space of action in the present through technical means. Only by accepting this reality will we be able to grasp the pharmacology of our contemporary media situation, of the general ecology at issue here: namely, that whatever recompense might come to us from the technical access to sensibility must come through the very operations that lie at the heart of today's data capitalism. Realizing any kind of pharmacological recompense involves giving up precisely what Thrift seems unable to give up: the hope for a direct phenomenological interface with the reengineered, preaffective present.

It is thus perfectly understandable that Thrift doesn't envisage the possibility for a different, "phenomenology-implicating" engagement with the operational present of sensibility, the very operation informing my intervention here: feed-forward. Rather than seeking to restore human sensory and perceptual agency within that present, as Thrift's various recipes for the creation of "neurophenomenological worlds" all do, the operation of feed-forward recognizes the radical opacity of this pre-conscious and pre-affective domain and utilizes the technical means developed by today's data industries to access it in order to feed it forward into a just-to come future experience of embodied consciousness. Whereas Thrift's recourse to instantaneous simulation mistakenly indulges the vain fantasy of human agency within the domain of braintime, feed-forward perfectly instantiates the "diagram" of the braintime experiment as it has been repeatedly performed in the laboratory since the 186os: feedforward brings very fast - and by definition, non-phenomenologizable - processes into the slower spacetime interval of durationally-embodied human experience. ${ }^{9}$

Let me conclude by drawing out some implications of my argument 
for media theory and the ecologization of media. In the wake of the ecological pharmacology of twenty-first century media, we must turn away from analyzing media as objectifications of the flux of mental experience or consciousness and seek to intervene in the far more indirect processes whereby media impacts the sensory ecology - worldly sensibility - on which such higher order experience emerges. Accordingly, in developing an account of mediological modulation - of the way media shapes the domain of sensibility prior to any perceptual experience of it - we must constantly bear in mind that we are not re-engineering the present to expand human agency within the space of that present, but are rather modulating a present we literally cannot live, in order to engineer experienceto-come in the future. What this means is that the impact of mediological modulation cannot be direct - in the sense that media effects would directly impact human sensations and perceptions - but must be indirect, ecological, and predictively anticipatory: such modulation intervenes at the level of worldly sensibility itself prior to - but, crucially, not without relation to - whatever affective, sensory and perceptual experience might emerge from it. Put another way, such modulation involves designing media environments out of the general ecology of media that channel pre-affective sensibility, itself beyond the access of direct human agency, in ways predictably likely to yield certain kinds of affective, sensory, and perceptual experiences. In sum: because the operational present of sensibility directly targeted by data capitalism lies outside the domain, and beyond the reach, of embodied perceptual and affective experience, the modulation of sensibility at issue here must follow the diagram of feed-forward: it must, that is, develop the potential for a mediological modulation of the domain of sensibility, general ecology, that would be "autonomous" from any direct connection to human affections, sensations, and perceptions and that would embrace the technicity "essential" to its very mode of being.

That is why nothing less is at stake here than the very status - indeed the autonomy - of media and the ecological networks it generates. For what lies at the heart of mediological modulation in the sense sketched above is an operation of media that takes place beneath all phenomenological horizons, including the "neurophenomenological" ones, and that is radically disjoined from any immediate human agency or access. Exploring the potentiality for mediological modulation of sensibility will accordingly require us to develop a non-anthropomorphic, non-phenomenological, non-prosthetic, and radically ecological theory of media. 
The Operational Present of Sensibility

\section{Notes}

1. Jordan Crandall, "The Geospatialization of Calculative Operations: Tracking, Sensing and Megacities," Theory, Culture \& Society 27, no. 6 (2010): 68-90, 75.

2. Crandall, "The Geospatialization of Calculative Operations," 87-88.

3. Nigel Thrift, Non-Representational Theory: Space, Politics, Affect (New York: Routledge, 2007), 36-37.

4. Henning Schmidgen, "Lecture in the Mellon Sawyer Seminar on Phenomenology Between Mind and Media," Duke University, April 19, 2012, audiofile available at http://phenomenologymindsmedia.wordpress.com/. See also Schmidgen, "The Donders Machine: Matter, Signs, and Time in a Physiological Experiment, ca. 1865," Configurations 13 (2005): 211-56.

5. Catherine Malabou, The New Wounded: From Neurosis to Brain Damage (New York: Fordham University Press, 2012), 42.

6. Malabou, The New Wounded, 43.

7. Schmidgen, "Lecture."

8. Crandall, "The Geospatialization of Calculative Operations," 85-86.

9. Schmidgen, "Lecture." 